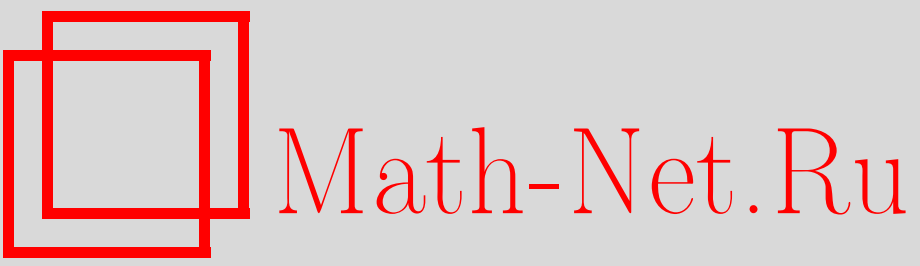

М. И. Зеликин, Асимптотика уклонения функционала от оптимального значения при замене четтеринга субоптимальным режимом, УМH, 1999, том 54, выпуск 3, 163-164

DOI: https://doi.org/10.4213/rm174

Использование Общероссийского математического портала Math-Net.Ru подразумевает, что вы прочитали и согласны с пользовательским соглашением

http://www. mathnet.ru/rus/agreement

Параметры загрузки :

IP: 52.23 .180 .231

26 апреля 2023 г., 17:00:07 


\title{
АСИМПТОТИКА УКЛОНЕНИЯ ФУНКЦИОНАЛА \\ ОТ ОПТИМАЛЬНОГО ЗНАЧЕНИЯ ПРИ ЗАМЕНЕ ЧЕТТЕРИНГА СУБОПТИМАЛЬНЫМ РЕЖКИМОМ
}

\author{
М.И. ЗЕЛикин, Л.Ф. ЗЕЛИКИнА
}

Под термином четтеринг-траектории понимаются такие оптимальные траектории, которые имеют бесконечное число переключений на конечном интервале времени. Многие экстремальные задачи прикладного содержания, такие как задачи оптимального управления движением роботов, задачи космической навигации, математической экономики, электротехники и др., имеют оптимальные четтеринг-траектории [1], [2]. Возникает вопрос о практической реализации этих траекторий. Каковы будут потери при аппроксимации четтеринг-траекторий траекториями с конечным числом переключений? Для ответа на этот вопрос рассмотрим случай, когда особой экстремалью является начало координат.

Рассматривается задача минимизации функционала

$$
J=\frac{1}{2} \int_{0}^{\infty} x_{1}^{2}(t) d t
$$

на множестве решений системы

(2) $\quad \dot{x}_{i}=x_{i+1}+f_{i}(x) \quad(i=1, \ldots, n-1), \quad \dot{x}_{n}=h_{0}+u h_{1}+f_{n}(x), \quad|u| \leqslant 1$.

Здесь $x \in \mathbb{R}^{n} ;$ функции $f_{i}: \mathbb{R}^{n} \rightarrow \mathbb{R}(i=1, \ldots, n)$ бесконечно дифференцируемы. Управления $u(t)$ измеримы; соответствуюшие траектории - $x(t)$ - абсолютно непрерывны.

Рассмотрим вспомогательную управляемую систему

$$
\dot{x}_{i}=x_{i+1}(i=1, \ldots, n-1), \quad \dot{x}_{n}=h_{0}+u h_{1}, \quad|u| \leqslant 1 .
$$

Система (3) инвариантна относительно действия однопараметрической группы преобразований $g_{\lambda} x=\left(\lambda^{n} x_{1}, \lambda^{n-1} x_{2}, \ldots, \lambda x_{n}\right) ; g_{\lambda} t=t / \lambda ; g_{\lambda} u=u(\lambda>0)$.

В пространстве $\mathbb{R}^{n}$ введем норму $\|x\|=\max _{i}\left|x_{i}\right|^{\frac{1}{n-i+1}}$. Шарами относительно этой нормы служит семейство параллелепипедов, инвариантное относительно действия группы $g_{\lambda}$, т.е. $\left\|g_{\lambda} x\right\|=\lambda\|x\|$.

ПреДПОЛОЖенИЕ 1. Существуют такие константы $r_{0}>0$ и $C>0$, что для любого $r \leqslant r_{0}$ справедливо неравенство $\max _{\|x\| \leqslant r}\left|f_{i}(x)\right| \leqslant C r^{n-i+1}$.

Предположение 1 означает, что система (3) является главной частью системы (2) относительно действия группы $g_{\lambda}$.

Рассмотрим функции $z_{i}(x, \psi)=\operatorname{ad}_{H_{0}}^{i} H_{1}(i=0, \ldots, 2 n-1)$. Здесь $\operatorname{ad}_{\Phi} F=\{\Phi, F\}-$ скобка Пуассона, которая определяет производную $\frac{d}{d t} F$ в силу гамильтоновой системы с гамильтонианом $\Phi$.

ПРЕДПОЛОЖЕНИЕ 2. Предположим, что начало координат является особой экстремалью задачи (1), (2) глобального порядка $n$ (см. [3]). Предположим, что функции $z_{i}$ $(i=0, \ldots, 2 n-1)$ функционально независимы в некоторой окрестности начала координат.

Принцип максимума Понтрягина для задачи (1), (2) сводится в переменных $z_{i}$ к виду

$$
\dot{z}_{i}=z_{i+1} \quad(i=1, \ldots, 2 n-1), \quad \dot{z}_{2 n}=\alpha(z)+\beta(z) \operatorname{sgn} z_{1} .
$$

ПРЕДПОЛОЖЕНИЕ 3. 1) На особой әкстремали $x=0$ выполнено обобщенное необходимое условие оптимальности Лежсандра-Клебша: $(-1)^{n-1} \beta(0)>0$.

2) Существует допустимое управление, удерживающее фазовую точку в начале координат: $|\alpha(0)|<|\beta(0)|$.

Работа выполнена при финансовой поддержке Российского фонда фундаментальных исследований (гранты № № 99-01-01224, 98-01-00535). 
Метрика в пространстве $\mathbb{R}^{2 n}$ определяется формулой $\|z\|_{1}=\max _{i}\left|z_{i}\right|^{\frac{1}{2 n-i+1}}$.

Субоптимальньй режим, содержащий конечное число переключений, строится следующим образом. Для начальной точки $x_{0}$ рассмотрим начальный участок оптимальной траектории $x^{*}(t)$, содержащий ровно $N$ переключений. Этот участок заканчивается в точке, которую мы обозначим $x_{N}$. Начиная с точки $x_{N}$, используем произвольное управление $\hat{u}(t)$, переводящее $x_{N}$ в начало координат за время $T\left(x_{N}\right)$, удовлетворяющее оценке $T\left(x_{N}\right) \leqslant A\left|x_{N}\right|$. В [4] доказано, что в качестве $\hat{u}(t)$ можно взять, например, оптимальное управление для задачи быстродействия в начало координат по траекториям линейной управляемой системы (3). Значение функционала (1) на оптимальной траектории с начальным значением $x$ обозначим через $J^{*}(x)$; значение функционала (1) на субоптимальной траектории с начальным значением $x$, содержащей $N$ оптимальных переключений, обозначим через $\hat{J}_{N}(x)$. Обозначим через $z_{x}^{*}(t)$ решение системы (4), отвечаюшее оптимальной траектории задачи $(1),(2)$ с начальным значением $x$. Пусть $\left\|z_{x}^{*}(0)\right\|_{1}=r$. Обозначим $Q\left(z_{x}^{*}(0)\right)=\inf \left\{t \geqslant 0 \mid\left\|z_{x}^{*}(t)\right\|_{1}=r / 2\right\}$ - время первого попадания образа оптимальной траектории в пространстве $\mathbb{R}^{2 n}$ на сферу вдвое меньшего радиуса.

Прежде чем формулировать основную теорему, сформулируем вспомогательные леммы.

Лемма 1. Существуют такие константы $r_{0}>0$ и $K>0$, что для любой оптимальной траектории с начальнымм данными $\left\|z_{x}^{*}(0)\right\|_{1}=r\left(r \leqslant r_{0}\right)$ имеет место оценка $\left\|z_{x}^{*}(t)\right\|_{1}<K r$ npu всех $t>0$.

Лемма 2. Существует такая константа $b>0$, что функиия $Q\left(z_{x}^{*}(0)\right)$ удовлетворяет оценке $Q\left(z_{x}^{*}(0)\right) \leqslant b r$.

Лемма 3. Пусть $z^{*}(t)$ - оптимальное решение задачи (1), (2), которое удовлетворяет гамильтоновой системе принципа максимума Понтрягина (4) с начальными условиями $\left\|z^{*}(0)\right\|_{1}=r\left(r \leqslant r_{0}\right)$. Тогда существует такое $a>0$, что на отрезке $0 \leqslant t \leqslant$ аг функиия $z_{1}^{*}(t)$ имеет не более чем $2 n$ корней, считая с их кратностью.

Теорема 1. Пусть выполнены предположения 1-3. Тогда существуют область $\Omega \ni 0$ и константы $M>0$ и $\mu>0$ такие, что для любого $x_{0} \in \Omega$ справедлива оценка

$$
\hat{J}_{N}(x)-J^{*}(x)<M e^{-\mu N} .
$$

СХемА ДОКАЗАТЕЛЬСтвА. Обозначим $m=([b / a]+1) 2 n$, где $[b / a]-$ целая часть числа $b / a$. Из лемм 2 и 3 следует, что на отрезке времени $0 \leqslant t \leqslant b r$ функция $z_{1}^{*}(t)$ имеет не больше чем $m$ корней, т.е. на этом отрезке произойдет не больше чем $m$ переключений. Следовательно, после $m$ переключений норма вектора $z$ уменьшится вдвое, а это означает, что норма вектора $x_{N}$ с ростом числа $N$ убывает как геометрическая прогрессия

Имеем

$$
\left|x_{N}\right| \leqslant L e^{-\gamma N} .
$$

$$
\hat{J}_{N}(x)-J^{*}(x)=\hat{J}_{0}\left(x_{N}\right)-J^{*}\left(x_{N}\right)=\int_{0}^{T\left(x_{N}\right)} x_{1}^{2} d t-J^{*}\left(x_{N}\right) .
$$

В силу леммы 1 функция $x_{1}(t)$ ограничена, поэтому интеграл в правой части $(7)$ не превосходит $C T\left(x_{N}\right)$. Используя (6), получаем искомую оценку (5), что и требовалось доказать.

Тем самым, при замене четтеринга субоптимальным режимом с конечным числом переключений уклонение функционала от его оптимального значения экспоненциально убывает с ростом числа использованных переключений.

\section{СПИСОК ЛИТЕРАТУРЫ}

[1] Зеликин М.И., Борисов В.Ф. // Труды МИАН. 1991. Т. 197. С. 85-166. [2] Zеlikin M., Borisov V. Theory of Chattering Control with Applications to Cosmonautics, Robotics, Economics, and Engineering. Boston: Birkhäuser, 1994. [3] Зеликин М. И. // Труды МИАН. 1999. Т. 224. [4] Манита Л. А. // Фунд. и прикл. матем. 1996. Т. 2. № 2. С. 411-447. 